\title{
Preliminary Evaluation of an Autologous Dendritic Cell Vaccine Using Nanoparticle Technology for the Treatment of Canine Malignant Melanoma
}

\author{
Meaghan Veronica Eren, Julianne Hwang, Janean Fidel, Rance Sellon, Cleverson de Souza* \\ Department of Veterinary Clinical Sciences, College of Veterinary Medicine, Washington State University, Pullman, Washington, USA
}

Email address:

cleverson.desouza@wsu.edu (C. D. Souza)

${ }^{*}$ Corresponding author

\section{To cite this article:}

Meaghan Veronica Eren, Julianne Hwang, Janean Fidel, Rance Sellon, Cleverson de Souza. Preliminary Evaluation of an Autologous Dendritic Cell Vaccine Using Nanoparticle Technology for the Treatment of Canine Malignant Melanoma. American Journal of Biomedical and Life Sciences. Vol. 9, No. 1, 2021, pp. 84-96. doi: 10.11648/j.ajbls.20210901.21

Received: February 3, 2021; Accepted: February 10, 2021; Published: February 23, 2021

\begin{abstract}
Canine malignant melanoma is an aggressive neoplasm that carries a poor prognosis due to its minimal responsiveness to traditional therapy protocols, particularly if the oral cavity, mucocutaneous junctions, or subungual sites are involved. This proof-of-concept study evaluated a prototype autologous dendritic cell vaccine using poly-lactic-co-glycolic (PLGA) nanoparticles containing antigens from patient-derived whole tumor lysate and the adjuvant monophosphoryl lipid A in five canines with stages III-IV malignant melanoma. Nanoparticle constructs biochemical characterization; encapsulation efficiency and kinetic release studies were determined. Our results showed that tumor antigens were successfully incorporated in the PLGA/monophosphoryl lipid A nanoparticle constructs. Additional in vitro experiments showed that the PLGA/monophosphoryl lipid A nanoparticle constructs effectively activated autologous dendritic cells, and generated a greater than twofold increase in the release of the pro-immune cytokine IFN- $\gamma$. No significant adverse effects were observed in any of the patients following intradermal vaccination, and flow cytometry of whole blood revealed increased CD4:CD8 T lymphocyte ratios by the completion of the study. These results suggest that a dendritic cell vaccine utilizing PLGA/monophosphoryl lipid A nanoparticle technology could potentially initiate an adaptive immune response and is safe to administer to canine patients. Further in vivo studies with a larger cohort of patients are warranted.
\end{abstract}

Keywords: Melanoma, Nanoparticles, Dendritic Cells, Adaptive Immunity, Cancer Vaccines

\section{Introduction}

Canine malignant melanoma (MM) is an aggressive neoplasm that carries a poor prognosis if the oral cavity, mucocutaneous junctions, or subungual sites are involved [3, 5]. MM is locally invasive and highly metastatic to regional, draining lymph nodes, liver, lungs, and adrenal glands [40]. In addition to the anatomic location of the primary neoplasm and the presence of metastasis, prognosis is linked to the size of the tumor. Based on the World Health Organization (WHO) staging system, dogs with primary tumors less than $2 \mathrm{~cm}$ in diameter (Stage I) have a better prognosis than dogs with tumors that range from $2-4 \mathrm{~cm}$ in diameter (Stage II) or greater than $4 \mathrm{~cm}$ in diameter (Stage III-IV) [40].

No "standard of care" protocols other than surgical resection have been established in veterinary medicine, as no consistent benefit of adjunctive therapy has been proven [1]. Although radiation therapy has been used to control local recurrence of MM, optimal protocols have not been identified, and median progression free survival (PFS) is dependent on stage of disease and tumor location [40]. For dogs with metastasis, palliative and conventional radiation therapy achieves median survival times (MSTs) of only 7 months [20, 40]. The addition or sole use of systemic chemotherapy has also proven to be ineffective, as response rates are generally low, and no significant impact on survival time has been shown $[4,21,40]$.

Due to minimal responsiveness to standard therapies, MM has garnered interest within the field of immunotherapy, and efforts have been made to target the patient's innate and/or 
adaptive immune systems through the use of individualized vaccines. The most recent breakthrough in treatment of MM was the release of a xenogeneic plasmid DNA vaccine with an insert encoding the full length human tyrosinase gene $[1,3$, 12]. Oncept ${ }^{(B)}$ (Merial, USA), the first USDA-licensed vaccine for the treatment of canine melanoma, generates a humoral response in dogs 3-9 months following completion of a biweekly protocol, while inducing minimal, mainly cosmetic side effects (i.e. vitiligo) [36]. It also results in a strong IFN- $\gamma$ response, resulting in T cell-mediated immunity [1]. Although a Phase I/II clinical trial concluded that Oncept ${ }^{\circledR}$ significantly improved MST for dogs with stage II or III, locally controlled oral MM, the limitations of the study design, the use of historical control populations and the degree of censoring employed for statistical analysis, raised questions regarding the validity of that claim $[1,12]$.

Compounding the controversy surrounding the Oncept ${ }^{\circledR}$ vaccine is a report that tyrosinase is detected via immunohistochemistry in only a small subset of canine patients with MM [27, 33]. This study, which calls the efficacy of a tyrosinase-specific vaccine into question, highlights the inherent ability of this neoplasm to avoid detection by the immune system. In addition to downregulating tyrosinase expression, MM can impair antineoplastic immune responses through the secretion of antiinflammatory cytokines (i.e. interleukin (IL)-10), the promotion of a T-regulatory cell response, and the alteration of antigen presenting cell (APC) function [1]. Due to the immune-suppressive tumor microenvironment, there is a critical need for an adjunctive therapy that can enhance cytotoxic $\mathrm{T}$ lymphocyte (CTL) responses and decrease immunosuppression within affected patients.

Research and clinical trials involving cases of human malignant melanoma have concentrated on vaccinations involving dendritic cells (DCs) due to their unsurpassed ability to orchestrate immune responses [29, 31, 35]. Treatment protocols have involved various means of stimulating DCs, including synthetic and natural peptidepulsed DCs, autologous or allogeneic tumor or tumor-lysate pulsed DCs, and mRNA-transduced DCs [24]. Although peptide pulsed-DCs have shown the most promise in terms of clinical response, the overall efficacy of DC-vaccines has been suboptimal thus far $[10,24]$. Poor efficacy is due to several inherent challenges, including the short duration of MHC class I/peptide/ $\beta 2$-microglobulin complex expression on the surface of DCs, inefficient cross-presentation of antigen to CTLs, and the elicitation of humoral rather than CTL responses [10, 28, 38, 39]. Underwhelming results are also likely due to uncertainties regarding the most effective route of DC-vaccine administration, poor in vivo stability of formulations and the insufficient immunogenicity of most tumor-associated antigens $[9,10,24]$.

To address these challenges, nanoparticulate carrier systems, including dendrimers and quantum dots, have been investigated [19]. When utilized as vehicles for antigen encapsulation, nanoparticles (NPs) have been shown to improve overall antigen uptake, processing, and presentation by DCs, thereby enhancing adaptive immune responses [35]. Poly (D,L-lactic-co-glycolic acid) (PLGA) NPs are of particular interest due to their minimal systemic toxicity and previous approval by the United States Food and Drug Administration (FDA) and European Medicine Agency (EMA) for use in vaccine delivery systems [8]. PLGA NPs can successfully encapsulate a variety of antigens (e.g. peptides; cell lysates), enhance the maturation of DCs and promote the cross-presentation of exogenous antigens via MHC class I pathways [8, 19, 39]. In addition, due to the slow hydrolysis of PLGA NPs to their constituent monomers (lactic acid and glycolic acid), antigen-specific CTLs can be stimulated for up to 9 days following interaction with nanoparticle-loaded DCs [39].

To further enhance the immunogenicity of this delivery system, adjuvants (i.e. bacterial lipid A analogs) have been incorporated into the NP formulations [32]. Monophosphoryl lipid A (MPLA), a modified, nontoxic derivative of lipopolysaccharide and a specific ligand of toll-like receptor 4 (TLR4), is currently one of the most promising adjuvants in nanomedicine [9]. Coencapsulation of adjuvant and antigen has proven to enhance immunotherapy strategies in a variety of species by enhancing the uptake of NP by DCs, enhancing DC maturation and promoting the secretion of proinflammatory cytokines (i.e. IL-2; IL-6; TNF- $\alpha$ ) [9, 15]. Through the enhanced secretion of these cytokines, T-cell proliferation and the induction of a potent $\mathrm{CD} 4+\mathrm{Th} 1$ profile can be generated. It is theorized that NP formulations involving MPLA can generate CTL responses in vivo, and initiate CD8+ T-cell memory [9].

Although studied in mice and humans, the use of PLGA NPs containing MPLA had not been evaluated in canines until 2016, at which time our group characterized the safety and effects of multiple NP constructs on canine macrophages in vitro. Our results indicated that canine macrophages incubated with NP formulations containing MPLA expressed significantly greater amounts of pro-inflammatory cytokines (TNF- $\alpha$ and IL-12p40) than those without the adjuvant [13]. In addition, safety was proven by demonstrating macrophage viability after incubation with NP constructs. Ultimately, the results of this study paved the way for further investigations into the use of PLGA NP containing MPLA in canines.

We sought to create an individualized form of immunotherapy utilizing PLGA NPs containing antigens from a whole tumor lysate (WTL) and MPLA. A WTL, comprised of undefined tumor-associated antigens, was selected in order to generate a broad array of antigenic epitopes. We hypothesized that antitumor CTL responses could be generated in canine patients with $\mathrm{MM}$, following successful ex vivo stimulation of autologous DCs with NP constructs. We report the safety, immune responses and clinical outcomes in 5 canines with MM. Initial experiments detailing lysate preparation, nanoparticle synthesis and characterization, autologous DC preparation and characterization, and cytokine expression following in vitro co-culture assays are also reported. To the authors' knowledge, this is the first report involving DC vaccination 
in canine MM patients that utilizes PLGA NP containing MPLA.

\section{Materials and Methods}

\subsection{Animals}

Five adult canines with stage III or IV oral MM, and a physical tumor $\geq 1 \mathrm{~cm}$ in diameter were enrolled for the in vivo portion of this study after obtaining signed, informed consent from the owners. The demographics of the enrolled canines and all prior treatments for $\mathrm{MM}$ are presented in Table 1. All patients were $\geq 15 \mathrm{~kg}$. MM was diagnosed following cytologic and/or histopathologic evaluation of the tumor by a board-certified clinical or anatomic pathologist via fine-needle aspiration or biopsy, respectively. Immunohistochemistry involving antibodies against Melan-A, PNL2, TRP-1 and TRP-2 (Michigan State University Diagnostic Center for Population \& Animal Health) was required for diagnosis in one patient (Patient 4) who presented with a spindle-shaped variant of an amelanotic melanoma. At the time of enrollment, 4 out of 5 patients were receiving an oral antibiotic to control secondary infection of the tumor and halitosis. This study was approved by the Institutional Animal Care and Use Committee (IACUC) at Washington State University (protocol number 04834001/2016).

Table 1. Demographics of the dogs enrolled for the in vivo portion of the study.

\begin{tabular}{|c|c|c|c|c|c|c|}
\hline Patient No. & Sex & Breed (n) & Prior Treatment & Age (years) & Stage $^{d}$ & Site of Metastasis \\
\hline 1 & $\mathrm{FS}^{\mathrm{a}}$ & Poodle Mix & Surgical excision, RT, Oncept ${ }^{\circledR}$ & 13 & III & Lymph node \\
\hline 2 & $\mathrm{MN}^{\mathrm{b}}$ & Standard Poodle & $\mathrm{RT}^{\mathrm{c}}$ & 14 & IV & Lungs \\
\hline 3 & M & Blue Tick Coonhound & none & 12 & III & LNN \\
\hline 4 & FS & Labrador Retriever & none & 12 & III & \\
\hline 5 & $\mathrm{MN}$ & Chow Chow & RT & 9 & IV & Skin \\
\hline
\end{tabular}

${ }^{\mathrm{a}} \mathrm{FS}=$ female spayed; ${ }^{\mathrm{b}} \mathrm{MN}=$ male neutered

${ }^{\mathrm{c}} \mathrm{RT}=$ radiation therapy, consisting of 3 fractions of 8 Gy over 3 weeks

${ }^{\mathrm{e}}$ Stage of disease is determined based on the World Health Organization (WHO) classification. Stage I disease includes patients with tumors $<2$ cm in their greatest dimension; stage II tumors are between 2 and $4 \mathrm{~cm}$ in diameter; stage III tumors are $>4 \mathrm{~cm}$ in diameter and/or have lymph node metastases; stage IV tumors have radiographically detectable lung metastases.

Two female spayed, Golden Retrievers between the ages of 1.5 and 2 years of age were enrolled to provide whole blood for in vitro experiments. The dogs were in good health, up to date on vaccinations and not on any medications apart from monthly ectoparasitic preventatives (K9 Advantix ${ }^{\circledR} \mathrm{II}$; Bayer, USA). A consent form was signed by the owners. Collection of canine blood for in vitro experiments was previously approved by IACUC in association with another study (protocol number 04548-001/2015).

\subsection{Generation of Immature Dendritic Cells}

Twenty $\mathrm{mL}$ of whole blood was collected from each patient with MM once a week for a total of 4 collections for the in vivo portion of this study. Sixty $\mathrm{mL}$ of whole blood was collected from 2 female spayed, Golden Retrievers once for the in vitro portion of this study. Whole blood was collected from the jugular vein using a $22 \mathrm{G}$ butterfly and syringe apparatus into $10 \mathrm{~mL}$ tubes containing ethylenediaminetetraacetic acid (EDTA). Whole blood was refrigerated at $4^{\circ} \mathrm{C}$ until processing. Ficoll-Paque ${ }^{\mathrm{TM}}$ Premium 1.084 (GE Healthcare, USA) was used to isolate mononuclear cells. Mononuclear cells were subsequently incubated with anti-mouse IgG CD14 magnetic cell sorting (MACS) microbeads with strong cross-reactivity with canine cells (MACS; Miltenyi Biotec, Germany). Isolated CD14+ monocytes were counted via an automated cell counter (Moxi Z Mini Automated Cell Counter; Orflo, USA) and suspended in complete RPMI medium containing $27.5 \mathrm{~mL}$ heat inactivated fetal bovine serum (Atlanta Biologicals, USA) and $2.7 \mathrm{~mL}$ Penicillin-Streptomycin (stock solutions 10,000 $\mathrm{U} / \mathrm{mL}$ Penicillin and 10,000 $\mu \mathrm{g} / \mathrm{mL}$ Streptomycin; Gibco, USA). Mononuclear cells were distributed into 6 well-culture plates $\left(1 \times 10^{6}\right.$ cells in $3 \mathrm{~mL}$ medium per well). Fifty $\mu \mathrm{g} / \mathrm{mL}$ each of canine recombinant GM-CSF (R\&D Systems, USA) and interleukin-4 (R\&D Systems, USA) were added to each well for the generation of immature DCs. Canine monocytes were incubated for 6 days at $37^{\circ} \mathrm{C}$ and $5 \% \mathrm{CO}_{2}$.

\subsection{Preparation of Whole Tumor Lysate (WTL)}

While under general anesthesia, approximately 1 gram of tissue was surgically excised from the patients' oral tumors and placed in $50 \mathrm{~mL}$ tubes until processing, which occurred within 2-4 hours. The tissue was cut into fine pieces, suspended in $1 \mathrm{~mL}$ cold PBS, and subjected to 4 freeze-thaw cycles involving liquid nitrogen and a $37^{\circ} \mathrm{C}$ warm water bath. Whole tumor lysates (WTLs) were then centrifuged at 15,400 $\times \mathrm{g}$ and $4^{\circ} \mathrm{C}$ for 15 minutes to remove cell debris as recommended by previous studies [28, 34]. Supernatants were collected and stored at $-30^{\circ} \mathrm{C}$ until further use. Individual protein content was determined using a Pierce ${ }^{\mathrm{TM}}$ BCA Protein Assay Kit and Genesys 20 spectrophotometer (both obtained from Thermo Fisher Scientific, USA). Supernatants were run on precast SDS-PAGE electrophoretic gels, which were subsequently stained with Coomassie Brilliant Blue.

\subsection{Nanoparticle Generation}

PLGA NP containing MPLA and antigens from WTL were generated using a double emulsion $\mathrm{W} / \mathrm{O} / \mathrm{W}$ technique that has been previously described [13]. Following dissolution of $9 \mathrm{mg}$ PLGA 75:25 (PolySciTech, USA) in $450 \mu \mathrm{L}$ of chloroform, $50 \mu \mathrm{L}$ of WTL (diluted to $20 \mathrm{mg} / \mathrm{mL}$ in PBS buffer), and $50 \mu \mathrm{L}$ of $2 \mathrm{mg} / \mathrm{mL}$ vaccine grade MPLA 
(InvivoGen, USA) were added to the solution. The solution was sonicated twice (30 seconds each) at an amplitude of $20 \%$ using a Branson Ultrasonics Sonifier ${ }^{\mathrm{TM}}$ Cup Horn sonicator. The emulsion was added to $1 \mathrm{~mL}$ of $1 \%$ polyvinyl alcohol (Sigma Aldrich, USA) while being vortexed, and subsequently sonicated 3 additional times (40 seconds each) at an amplitude of $40 \%$. The emulsion was then added dropwise into $5 \mathrm{~mL}$ of $1 \%$ polyvinyl alcohol and continuously stirred for 4 hours to promote evaporation of the chloroform. After that period, the mixture was centrifuged at $17,000 \times \mathrm{g}$ for 15 minutes at $4^{\circ} \mathrm{C}$ and washed 3 times using $1.5 \mathrm{~mL}$ of MilliQ water. After the final washing, $1 \mathrm{~mL}$ of MilliQ water was added to the pellet of PLGA nanoparticle constructs. The pellet was resuspended via bath sonication and stored at $-20^{\circ} \mathrm{C}$ until the sample could be freeze-dried using a Cressington Deep Temp Freeze Dryer (maintained by the WSU Franceschi Microscopy \& Imaging Center).

\subsection{Nanoparticle Characterization}

Hydrodynamic diameter, zeta potential, polydispersity index, mobility and conductivity of the NPs were assessed by nanoComposix, Analytical Services in San Diego, CA. To determine the extent of protein encapsulation, $2.4 \mathrm{mg}$ of PLGA/MPLA/WTL NPs were dissolved in $0.4 \mathrm{~mL}$ of $0.1 \mathrm{M}$ $\mathrm{NaOH}$ solution, and the protein within the supernatant was quantified using the Pierce ${ }^{\mathrm{TM}}$ BCA Protein Assay Kit (ThermoFisher Scientific, USA) after a 24-hour incubation period at $37^{\circ} \mathrm{C}$ with continuous agitation in an orbital shaker. Samples were centrifuged prior to analysis. Encapsulation efficiency was determined using a standard formula [28]:

(Mass protein extracted/Mass protein used in the encapsulation procedure) $\times 100$

To determine the release of protein from the NP constructs, $1 \mathrm{mg}$ of PLGA/MPLA/WTL NP was suspended in $1 \mathrm{~mL}$ of PBS and incubated at $37^{\circ} \mathrm{C}$ with continuous agitation in an orbital shaker. At specified time points (i.e. every 24 hours for 7 days, then once a week for a total of 3 weeks), the suspension was centrifuged, and the supernatant was collected and stored at $-30^{\circ} \mathrm{C}$ until further analysis. The original pellet was resuspended in $1 \mathrm{~mL}$ of PBS following collection of the supernatant. Protein within the supernatant was quantified using the Pierce ${ }^{\mathrm{TM}}$ BCA Protein Assay Kit.

\subsection{Evaluation of Dendritic Cell Maturation}

Following 6 days of culture, immature DCs were incubated with $150 \mu \mathrm{L}$ of PLGA/MPLA/WTL NPs $(1 \mathrm{mg} / \mathrm{mL})$ for 24 hours. After this period, the DCs were harvested using a Corning ${ }^{\mathrm{TM}}$ Falcon $^{\mathrm{TM}}$ cell scraper with a $1.8 \mathrm{~cm}$ blade, washed twice and suspended in DPBS. Cells were immediately labeled in separate tubes containing crossreactive, unconjugated monoclonal antibodies (moAbs) directed against MHC I (DG-H58A, Monoclonal Antibody Center, WA) and MHC II (DG-TH14B, Monoclonal Antibody Center, WA), as well as cross-reactive FITC antimouse CD80 (BioLegend, USA). Negative controls were incubated with a cross-reactive, unconjugated monoclonal IgG1 antibody against E.coli (COLIS69A, Monoclonal
Antibody Center, WA). All samples that utilized unconjugated moAbs were further incubated with $50 \mu \mathrm{L}$ of FITC goat anti-mouse $\mathrm{IgG} / \mathrm{IgM}$ diluted 1:200 in PBS (BD Pharmigen, USA). Samples were run on a BD FACS Calibur $^{\mathrm{TM}}$ and analyzed using FCS Express 4. Results were plotted as histograms against DCs that had not been cultured with PLGA/MPLA/WTL NPs.

\subsection{Vaccine Generation and Administration}

After a 24-hour incubation period with PLGA/MPLA/WTL NPs, DCs were collected using a cell scraper, washed with PBS, and centrifuged at $500 \times \mathrm{g}$ for 5 minutes. The supernatant was discarded, and the pellet was resuspended using $0.25 \mathrm{~mL}$ PBS and bath sonication. The suspension was collected into a sterile $1 \mathrm{~mL}$ syringe and maintained at $4^{\circ} \mathrm{C}$ until the patient's vaccination appointment with the WSU Oncology Service. The vaccine, which contained an average of $1 \times 10^{6} \mathrm{DCs}$ (range $=6.5 \times 10^{5}$ to 2.5 $\times 10^{6}$ ), was refrigerated for no longer than 45 minutes prior to administration. The patient received a complete physical examination prior to vaccine administration, as well as a complete blood count (CBC), biochemistry panel and urinalysis. All laboratory data was obtained from the WSU Clinical Pathology Laboratory. The vaccine was administered intradermally within the shoulder region. The patient was monitored within the WSU Veterinary Teaching Hospital (VTH), and vital parameters were recorded every 30 minutes. Adverse reactions, as outlined by the National Cancer Institute's Common Terminology Criteria for Adverse Events (CTCAE) v. 4.0, were graded and recorded [2]. The vaccine was administered once a week for a total of 4 intradermal vaccinations. Three-view thoracic radiographs and fineneedle aspirates of local, draining lymph nodes were performed at the beginning and end of the study.

\subsection{Flow Cytometry of Blood Lymphocytes}

Three $\mathrm{mL}$ of whole blood was collected in EDTA at the beginning and end of the vaccination series to assess circulating lymphocyte populations via flow cytometry. To distinguish between CD4+ and CD8+ lymphocytes, unconjugated, anti-canine moAbs specific for CD4 (DH29A, Monoclonal Antibody Center, WA) and CD8 (CADO46, Monoclonal Antibody Center, WA) were utilized, in addition to moAbs against CD5 (DH3B, Monoclonal Antibody Center WA) and CD21 (P. Moore, University of California Davis, CA). Use of a goat anti-mouse secondary antibody was required (diluted 1:200). Samples were run on a BD FACS Calibur $^{\mathrm{TM}}$ and analyzed using FCS Express 4.

\subsection{Dendritic Cell/T Cell Co-culture Studies}

All steps of this process are diagrammed in Figure 1. CD14+ depleted mononuclear cells (lymphocytes) were collected during the magnetic cell sorting process in a sterile container following elution from the magnetic column. Cells were counted and suspended in complete RPMI medium, as described above. Lymphocytes were distributed into 6 wellculture plates $\left(1 \times 10^{6}\right.$ cells in $3 \mathrm{~mL}$ medium per well). For 
the first round of stimulation, six wells were incubated with either $50 \mu \mathrm{L}$ of diluted whole tumor lysate (WTL) from Patient \#5, $50 \mu \mathrm{g} / \mathrm{mL}$ PLGA/MPLA/WTL NPs, or Concanavalin A (ConA) $5 \mu \mathrm{g} / \mathrm{mL}$. Three wells were used as a negative control, and therefore, only PBS was added to the RPMI medium. The ConA was a gift from the Monoclonal Antibody Center in Pullman, WA. Lymphocytes were incubated for 6 days at $37^{\circ} \mathrm{C}$ and $5 \% \mathrm{CO}_{2}$.

Following 6 days of incubation, immature DCs were incubated for 3 hours with either $50 \mu \mathrm{L}$ of diluted WTL from
Patient \#5, $50 \mu \mathrm{g} / \mathrm{mL}$ PLGA/MPLA/WTL NPs, or ConA 5 $\mu \mathrm{g} / \mathrm{mL}$. Three wells were used for each sample, and three wells were used as a negative control. Non-adherent lymphocytes were subsequently added to their corresponding wells of DCs and allowed to co-culture for an additional 6 days at $37^{\circ} \mathrm{C}$ and $5 \% \mathrm{CO}_{2}$. After 6 days, supernatants were collected and stored at $-30^{\circ} \mathrm{C}$ for future analysis. All cells were harvested by using a cell scraper, washed twice, and suspended in DPBS for immediate cell-labeling and flow cytometric analysis.

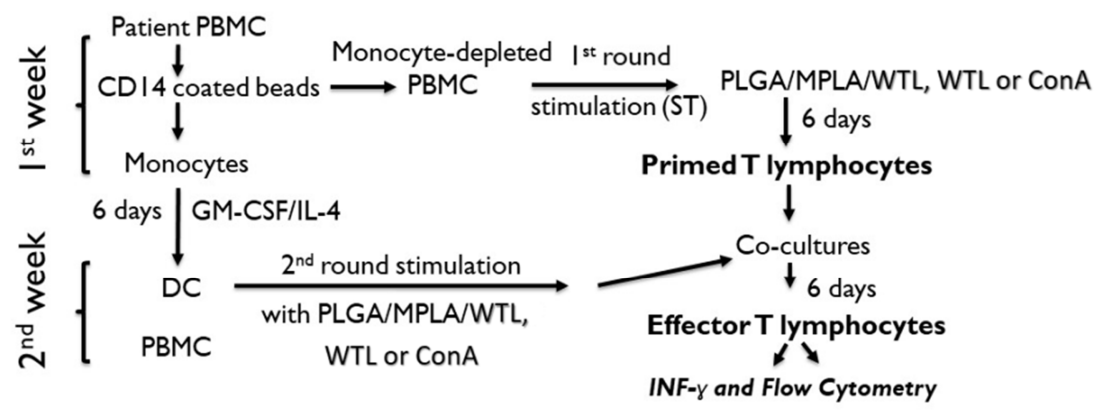

Figure 1. Steps of the dendritic cell/T cell co-culture studies.

\subsection{IFN-y Assay}

Cytokine profiling following dendritic cell/T cell coculture was performed on supernatants using Quantikine ${ }^{\circledR}$ ELISA for canine IFN- $\gamma$ (R\&D Systems, MN) as per manufacturer's instructions. The absorbance of the samples was measured within 30 minutes at $450 \mathrm{~nm}$ using a microplate reader (BioTek Instruments, USA). Supernatants from dendritic cell/ $\mathrm{T}$ cell co-cultures that had not been exposed to PLGA/MPLA/WTL NPs, WTL or ConA were used as negative controls.

\subsection{Flow Cytometry of Lymphocytes from DC/T Cell co-Culture}

Analysis of lymphocytes was performed using flow cytometry immediately after cell isolation (Day 0) and following completion of the dendritic cell/T cell co-culture period (Day 12). Briefly, the cells were incubated for 15 minutes with $10 \mu \mathrm{L}$ each of rat anti-dog CD4 (IgG) conjugated to Alex Fluor ${ }^{\circledR} 488$ (BioRad, USA), rat anti-dog CD8 (IgG) conjugated to Alex Fluor ${ }^{\circledR} 647$ (BioRad, USA), and mouse anti dog CD25 (IgG) conjugated to R. Phycoerythrin (RPE) (BioRad, USA), as per manufacturer's directions. Negative controls were incubated with an unconjugated moAb against COLIS69A, as previously described under "Assessment of Dendritic Cell Maturation." Samples were run on a BD FACS Calibur ${ }^{\mathrm{TM}}$ and analyzed using FCS Express4.

\subsection{Statistics}

Two-tailed, paired t-tests were used to compare data. Values of $\mathrm{p}<0.05$ were considered statistically significant. All analyses were performed using Excel 2016.

\section{Results}

\subsection{Characterization of the Whole Tumor Lysate}

Repetitive freeze-thaw cycles resulted in the release of highly variable concentrations of proteins from a standardized amount of tumor tissue (Table 2). An average of $69.2 \mathrm{mg} / \mathrm{mL}$ of protein was released, with a standard deviation of 19.7. Electrophoretic profiles, as determined by SDS-PAGE revealed similar protein banding patterns from all individual tumors, with prominent bands observed at 15,45 , and $70 \mathrm{kDa}$ for 3 of the sampled lysates (Figure 2). The distribution of protein bands exhibited banding patterns similar to that described by Prasad, et al [28]. These findings suggest that the repetitive freeze-thaw cycles result in the release of proteins and peptides of a similar molecular weight across all individual tumors.

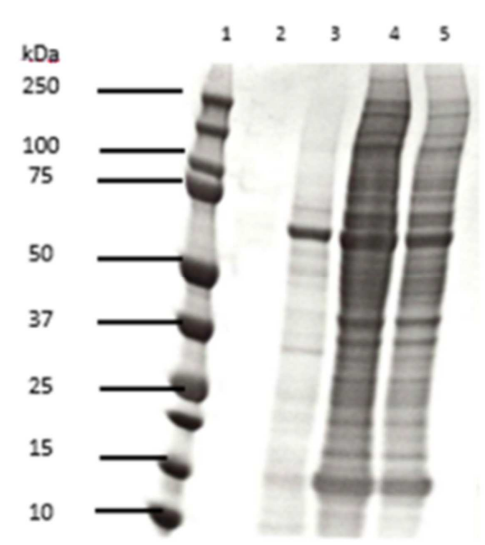

Figure 2. Electrophoretic profiles of WTLs from 3 patients, as determined by SDS-PAGE. Samples were collected as described in Material and Methods, and gels were stained with Coomassie Brilliant Blue. Prominent banding was noted at 15, 45, and $70 \mathrm{kDa}$. Lane 1, protein ladder; lane 2, empty; lane 3, WTL from Patient 1; lane 4, WTL from Patient 2; lane 5, WTL from Patient 3. 
Table 2. Protein concentration within the WTL as determined via the Pierce ${ }^{\mathrm{TM}}$ BCA Protein Assay Kit. Encapsulation efficiency and protein release after 21 days are also listed.

\begin{tabular}{llll}
\hline Patient No. & Protein concentration of WTL $\mathbf{( m g / m L )}$ & Encapsulation Efficiency $(\%)$ & Protein Released after 21 Days \\
\hline Patient 1 & 45.08 & 44.9 & $40.29 \mu \mathrm{g}(89.8 \%)$ \\
Patient 2 & 64.35 & 56 & $45.34 \mu \mathrm{g}(81 \%)$ \\
Patient 3 & 72.81 & 52.1 & $39.19 \mu \mathrm{g}(75.2 \%)$ \\
Patient 4 & 64.35 & 49.2 & $46.11 \mu \mathrm{g}(93.7 \%)$ \\
Patient 5 & 99.42 & 63.2 & $53.68 \mu \mathrm{g}(84.9 \%)$ \\
\hline
\end{tabular}

Repetitive-freeze thaw cycles resulted in the release in a variable amount of protein from a standard amount of tissue. An average of $69.2 \mathrm{mg} / \mathrm{mL}$ of protein was released, with a standard deviation of 19.7. EE ranged from 44.9-63.2\%, with an average of 53.08\% and standard deviation of 6.96. Between 75.2-93.7\% of the encapsulated proteins were released by the end of 21 days incubation period of PLGA/MPLA/WTL nanoparticles in $1 \mathrm{~mL}$ of PBS.

\subsection{Characterization of PLGA/MPLA/WTL NP Constructs}

Characterization of representative NP constructs from Patients 1 and 3 , as determined by nanoComposix, are listed in Table 3. The reported Result Quality field was acceptable for both samples, indicating that the data quality was high, and that there was confidence in its reliability. The mean diameters were 706.9 and $1085 \mathrm{~nm}$, respectively. The zeta potentials (electrokinetic potentials) were negative, ranging from -15.6 to $-12.5 \mathrm{mV}$, which are similar to the zeta potentials previously reported by our laboratory [13]. This confirms the good reproducibility, and stable formulations generated by the methods used, even when encapsulating novel, tumor-associated antigens. Polydispersity index ranged from $0.431-0.607$, indicating mild heterogeneity of the NPs.

Table 3. Data obtained from nanoComposix regarding representative nanoparticle constructs from Patients 1 and 3.

\begin{tabular}{llllll}
\hline Patient No. & Hydrodynamic Diameter (nm) & Zeta Potential (mV) & Polydispersity Index & Mobility (umcm/Vs) & Conductivity (mS/cm) \\
\hline Patient 1 & 72.81 & -15.6 & 0.607 & -1.224 & 18 \\
Patient 3 & 99.42 & -12.5 & 0.431 & -0.9785 & 17.2 \\
\hline
\end{tabular}

The zeta potentials are similar to those previously reported by our laboratory, which confirms the good reproducibility and stable formulations generated by the methods used, even when encapsulating novel, tumor associated antigens. Polydispersity index ranged from $0.431-0.607$, indicating mild heterogeneity of the nanoparticles.

The encapsulation efficiency (EE) for the PLGA/MPLA/WTL NPs ranged from 44.9-63.2\%, as demonstrated in Table 2. The average EE was $53.08 \%$ with a standard deviation of 6.96 . These percentages were comparable to those documented in previous studies that utilized lysates derived from rodent and human squamous cell carcinoma cell lines [28]. Claims of increasing EE by altering lysate concentration could not be substantiated, as all patient lysates were diluted to $20 \mathrm{mg} / \mathrm{mL}$ in PBS prior to encapsulation [28].

\subsection{Release Kinetics}

Supernatant samples were analyzed as described above to quantify the amount of protein released over a 3-week period (Figures 3a-b). This value represents the amount of antigen available for cross-presentation to lymphocytes. The maximum release of protein was noted on Day 4, as opposed to during the first 24 hours, which was previously reported $[28,34]$. The clinical relevance of this discrepancy is likely negligible since the approximate functional in vivo lifespan of an antigen-loaded DC is approximately 8 days [28]. Maximum release was followed by the release of small amounts of protein for the remaining time points. An average of $2.19 \mu \mathrm{g}$ and $1.58 \mu \mathrm{g}$ of protein per $1 \mathrm{mg}$ NP were released during Weeks 2 and 3, respectively. Although constant, the amount of protein was lower than previously reported by other investigators, including our own laboratory, during kinetic studies involving tumor-associated antigens and/or OVA $[13,28,34]$. This may be in part due to polymer shapes, and the ionic interactions between encapsulated proteins and
PLGA terminal end-groups [28]. Ultimately, $75.2 \%$ to $93.7 \%$ of the encapsulated proteins were released by the end of 21 days (Table 2).

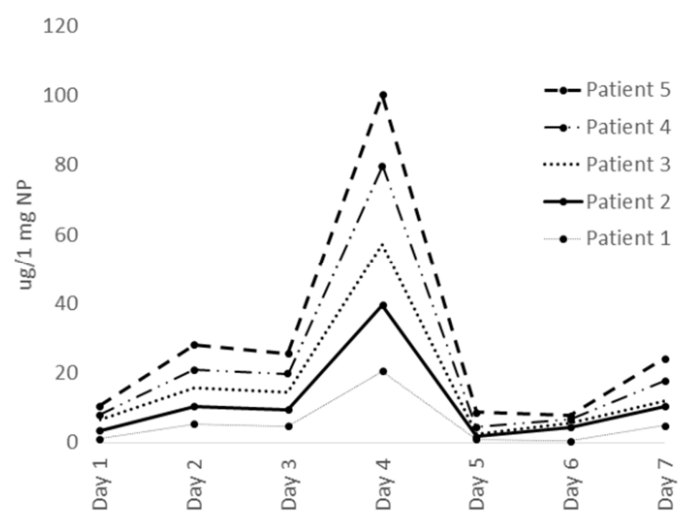

Figure 3. Protein release studies revealed maximum release on Day 4 in all patients.

\subsection{Evaluation of Dendritic Cell Phenotype and Flow Cytometric Profiles}

Following a 24-hour incubation period with PLGA/MPLA/WTL NPs, DCs were assessed using flow cytometry. Dendritic cells that had not been stimulated with NPs were used as a control and were considered immature. As shown in Figures 5-c, monocyte-derived DCs (MoDC) demonstrated increased expression of MHC I, MHC II and CD80 following stimulation with NP constructs. This finding, 
which was confirmed in 2 patients, is similar to a previously published study that demonstrated significantly higher MHCII and CD80 expression (along with CD40 and CD86) in MoDC stimulated with LPS [14]. This denotes successful maturation and activation of DCs, necessary for presentation of antigen to effector cells (lymphocytes) within lymph nodes.

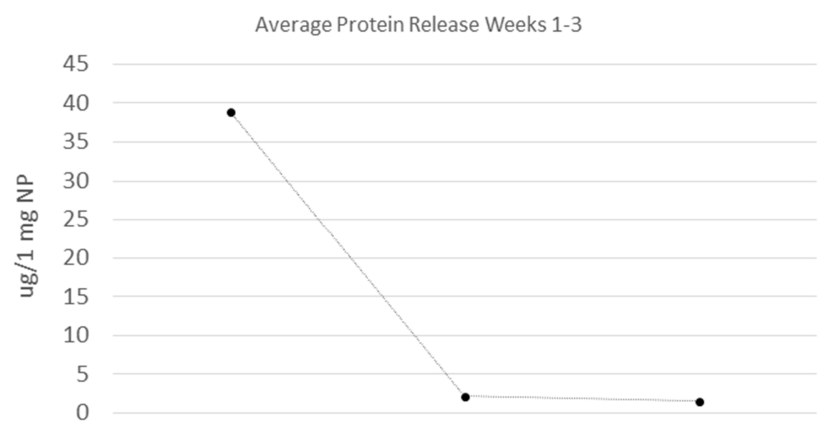

Figure 4. An average of $2.191 \mu \mathrm{g}$ and $1.578 \mu \mathrm{g}$ of protein was released during Weeks 2 and 3, respectively. $75.2 \%$ to $93.7 \%$ of the encapsulated proteins were released by the end of 21 days.

\subsection{Vaccine Administration and Patient Responses}

Intradermal vaccination consistently resulted in an approximately $1 \mathrm{~cm}$ bleb that regressed within 4 hours of administration. Three out of the 5 subjects $(60 \%)$ experienced at least one adverse event as outlined by the Common Terminology Criteria for Adverse Events v. 4.0 during the study (Table 4). All adverse events were considered mild (Grade 1) and resolved without intervention within a 24-hour period. Patient 5 demonstrated prolonged anorexia and expiratory stridor, which responded to an intramuscular injection of triamcinolone administered by the primary care veterinarian. These clinical signs are not included as adverse events, as they developed prior to initiation of immunotherapy, and were most likely due to the patient's thickened soft palate and stenotic oropharynx. The reported episode of diarrhea in Patient 1 was considered unrelated to the study, as it occurred following a diet change made by the owner and a scheduled trip to the groomer less than 24 hours following vaccine administration. Therefore, gastroenteritis and/or stress-induced colitis were strongly suspected.

Table 4. Summary of adverse events, as outlined by the CTCAE v. 4.0. All events were considered mild and resolved without medical intervention within a 24 hour period.

\begin{tabular}{|c|c|c|c|c|}
\hline Patient & Adverse Event & Grade & Comments & Reported Occurrence \\
\hline Patient 1 & Diarrhea & 1 & Mild increase in number of stools with mild change in consistency & Once following vaccines $\# 2 \& 3$ \\
\hline Detiont? & Lethargy & 1 & Lack of energy for 24-hour period & Once following vaccine \#1 \\
\hline Patient 2 & Injection site reaction & 1 & Warmth at the site of injection & Once following vaccines $\# 1 \& 2$ \\
\hline Patient 3 & N/A & & & \\
\hline $\begin{array}{l}\text { Patient } 4 \\
\text { Patient } 5\end{array}$ & $\begin{array}{l}\text { Injection site reaction } \\
\text { N/A }\end{array}$ & 1 & Pruritus at the site of injection & Once following vaccines $\# 1 \& 2$ \\
\hline
\end{tabular}

All vital parameters remained within normal limits during the monitoring period for all patients, and no signs of systemic toxicity were identified, as determined by serial hematologic and biochemical analyses. Mild fluctuations in serum glucose were appreciated, which were attributed to the stress associated with travel and hospitalization at the WSU VTH and/or alterations to the patient's normal feeding schedule on vaccination days. Patient 5 demonstrated a progressive, moderate neutrophilia with a variable left shift and monocytosis, attributed to inflammation associated with the primary tumor and/or the patient's previously diagnosed seborrheic dermatitis.

Three-view thoracic radiographs and fine-needle aspirates of submandibular lymph nodes were performed at the completion of the vaccination series and compared to preliminary data from the time of enrollment. In addition, tumor measurements were obtained by the veterinary oncologist in charge of each respective case. Following completion of the study, 4 patients demonstrated evidence of progressive disease, while 1 patient (patient 5) succumbed to disease prior to completion of the vaccination series. Two dogs (patients 3 and 4) experienced a $>25 \%$ increase in tumor size, while patient 1 developed pulmonary lesions suggestive of metastatic disease. Patient 2, previously diagnosed at the time of enrollment in the study with suspected pulmonary metastasis, demonstrated enlargement of several small, soft tissue nodules throughout the pulmonary parenchyma. These findings were interpreted as evidence of progressive disease by the attending radiologists. Lymph node aspirates, which confirmed metastatic disease prior to enrollment in 2 patients, remained relatively unchanged. The 1 subject without evidence of lymph node metastasis at the beginning of the study remained free of local and systemic metastasis at the end of the study. Overall MST following completion of the vaccine trial was 111 days (range $=56-167$ days).

\subsection{Characterization of Lymphocyte Populations in Whole Blood}

Flow cytometric analysis was performed on whole blood in order phenotype circulating lymphocyte populations, prior to and following completion of the vaccination series. Percentages and absolute numbers of CD4+, CD8+ and $\mathrm{B}$ lymphocytes are shown in Tables 5 and 6 . The total number of lymphocytes for each patient was obtained from a CBC performed the same day as the flow cytometric analysis. The absolute numbers of each subtype were calculated by multiplying the percentage of each cell type by the total number of lymphocytes. The ratios of CD4: CD8 (obtained by dividing the absolute number of CD4+ lymphocytes by the absolute number of CD8+ lymphocytes) are provided in Table 7. Data regarding post-vaccination lymphocyte populations are not available for Patient 5 since the patient succumbed to 
disease prior to completion of the vaccination series.

Table 5. Lymphocyte subpopulations in canine patients with Stage III-IV MM prior to initiation of immunotherapy. The total number of lymphocytes for each patient was obtained from a CBC performed the same day as the flow cytometric analysis. The absolute numbers of each subtype were calculated by multiplying the percentage of each cell type by the total number of lymphocytes.

\begin{tabular}{lllll}
\hline Parameter & Patient 1 & Patient 2 & Patient 3 & Patient 4 \\
\hline \%CD4+ T cells & 13.33 & 12.09 & 26.4 & 12.43 \\
\%CD8+T cells & 74.45 & 68.20 & 31.58 & 65.37 \\
\%B cells & 2.24 & 7.06 & 23.09 & 9.25 \\
Total lymphocytes (cells/uL) & 1026 & 1368 & 1639 & 936 \\
Total CD4+ T cells (cells/uL) & 136 & 165 & 433 & 8.02 \\
Total CD8+ T cells (cells/uL) & 763 & 933 & 518 & 612 \\
Total B cells (cells/uL) & 23 & 96 & 378 & 87 \\
CD4:CD8 ratio & 0.18 & 0.18 & 0.84 & 0.19 \\
\hline
\end{tabular}

Table 6. Lymphocyte subpopulations in canine patients with Stage III-IV MM on the final day of vaccination. Total numbers of lymphocytes were obtained as described under Table 5.

\begin{tabular}{lllll}
\hline Parameter & Patient 1 & Patient 2 & Patient 3 & Patient 4 \\
\hline \%CD4+ T cells & 3.00 & 21.61 & 41.85 & Patient 5 \\
\%CD8+T cells & 81.72 & 61.46 & 21.80 & - - \\
\%B cells & 2.88 & 10.62 & 15.77 & 29.90 \\
Total lymphocytes (cells/uL) & 1750 & 1104 & 102 & 2.93 \\
Total CD4+ T cells (cells/uL) & 53 & 239 & 43 & 539 \\
Total CD8+ T cells (cells/uL) & 1430 & 679 & 22 & 55 \\
Total B cells (cells/uL) & 50 & 117 & 16 & 161 \\
CD4:CD8 ratio & 0.04 & 0.35 & 2.00 & -- \\
\hline
\end{tabular}

Table 7. CD4:CD8 ratios pre- and post-vaccination. Although not statistically significant $(p=0.323)$, mild to moderate increases in CD4:CD8 ratios were observed.

\begin{tabular}{lll}
\hline & Pre & Post \\
\hline Patient 1 & 0.18 & 0.04 \\
Patient 2 & 0.18 & 0.35 \\
Patient 3 & 0.84 & 2.00 \\
Patient 4 & 0.19 & 0.34 \\
Patient 5 & 0.50 & -- \\
\hline
\end{tabular}

Although the CD4:CD8 ratios increased in 3 out of 4 patients, and modest changes were appreciated in the percentages of the different lymphocyte subtypes, no significant differences were found between the absolute numbers of $\mathrm{CD} 4+$ and CD8+ lymphocytes, or CD4:CD8 ratios, pre- and post-vaccination ( $p>.05)$. The study's small sample size could have contributed to the overall lack of

A

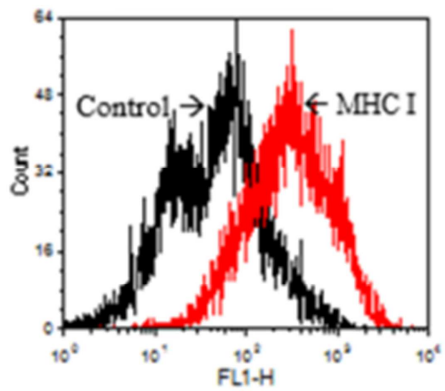

B

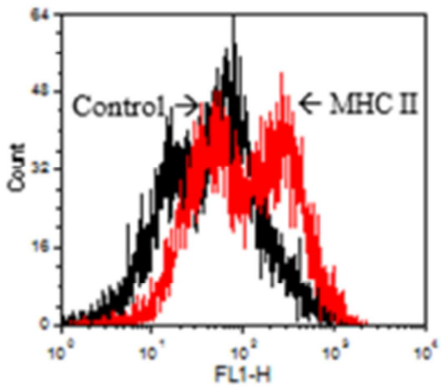

statistical significance. The mild to moderate increases in CD4:CD8 ratios, however, are still suggestive of proliferation of CD4+ lymphocytes, increased emigration of $\mathrm{CD} 8+\mathrm{T}$ lymphocytes into peripheral tissues and/or a selective decrease in CD8+ T lymphocytes. Selective decreases in both CD8+ $\mathrm{T}$ lymphocytes and $\mathrm{B}$ lymphocytes have been previously documented in canines with sarcomas, carcinomas, lymphomas, and mast cell tumors, and are attributed to the inhibitory effects of regulatory T cells (Tregs) [26]. Therefore, the possibility of cytotoxic lymphocyte suppression due to a hostile tumor microenvironment cannot be excluded. Egress of CD8+ lymphocytes into the tumor microenvironment, however, must also be considered, and warrants further exploration, as tumor-infiltrating lymphocytes have been linked with prognosis and survival rates in multiple forms of human and canine cancers [25].

Figure 5. Flow cytometric analysis of canine dendritic cells treated with PLGA/WTL/MPLA nanoparticles revealed increased expressed of surface markers MHC I (Figure 4a), MHC II (Figure 4b) and CD 80 (Figure 4c). One representative experiment is shown. Black histograms represent immature dendritic cells. Red histograms represent activated, mature dendritic cells that have been incubated with PLGA/MPLA/WTL nanoparticles for 24 hours. 


\subsection{IFN-y Assay}

IFN- $\gamma$ (a type II interferon that is synthesized and secreted predominantly by natural killer (NK) cells, CD4+ Th1 cells and $\mathrm{CD} 8+$ cytotoxic $\mathrm{T}$ cells) is a pro-inflammatory cytokine that promotes the activation of macrophages and induces MHC I and II expression [17]. Its expression was measured to assess the immunogenicity of PLGA/MPLA/WTL NPs following stimulation of lymphocytes by antigen-loaded, mature DCs. As shown in Figure 5, NP-mediated antigen delivery resulted in increased expression of IFN- $\gamma$ when compared against negative controls $(\mathrm{p}=0.159)$ and unencapsulated WTL $(\mathrm{p}=0.338)$. Although the lack of statistical significance is suspected to be the result of the small sample size that was evaluated $(\mathrm{n}=2)$, this finding needs to be confirmed using larger populations of dogs. This data suggests that tumor-associated antigens encapsulated within nanoparticle constructs decorated with MPLA could have a greater pro-inflammatory effect in vivo.

\subsection{Characterization of Lymphocytes in co-Culture}

Flow cytometric analysis of the lymphocytes in co-culture was performed to assess the phenotype responsible for the seemingly increased expression of IFN- $\gamma$ that was observed. Double-labeling of lymphocytes using conjugated moAbs against CD4, CD8 and CD25 demonstrated increased expression of the IL-2 receptor (CD25) following exposure to the positive control (ConA) in both subsets of lymphocytes. A representative sample is demonstrated in Figure 7a-d. Exposure to unencapsulated WTL resulted in a minimal increase in the expression of $\mathrm{CD} 25$ by $\mathrm{CD} 4+$ cells (up to $4.7 \%)$ and negligible expression of $\mathrm{CD} 25$ by $\mathrm{CD} 8+$ cells $(<$ $0.3 \%$ ). Exposure to PLGA/MPLA/WTL NPs resulted in a more robust response, with an increased percentage of CD4+CD25+ cells observed (Figure 7a-d). The expression of $\mathrm{CD} 25$ by CD $8+$ cells, however, was still negligible $(<1 \%)$.
Small populations of CD4- and CD8- cells that express CD25 were found in all co-culture studies in comparable proportions (Figure 7a-d). Absolute numbers cannot be provided since baseline peripheral blood evaluation were not performed prior to analysis.

The flow cytometry data fails to demonstrate significant activation of $\mathrm{CD} 4+$ and $\mathrm{CD} 8+\mathrm{T}$ cell subtypes following exposure to both tumor lysate and PLGA/MPLA/WTL NP formulations. The presence of a $\mathrm{CD} 25+$ double negative population of $T$ cells was unexpected. Since CD25 is not a specific marker for T-cell activation, the observed double negative cells may therefore represent activated B-cells, NK cells, or DCs [23]. Additional flow cytometric analyses using markers such as CD21, CD79a, and CD56 would be necessary to further characterize this population. The use of intracellular FoxP3 would also be necessary to further elucidate the identity of the $\mathrm{CD} 4+\mathrm{CD} 25+$ lymphocytes observed.

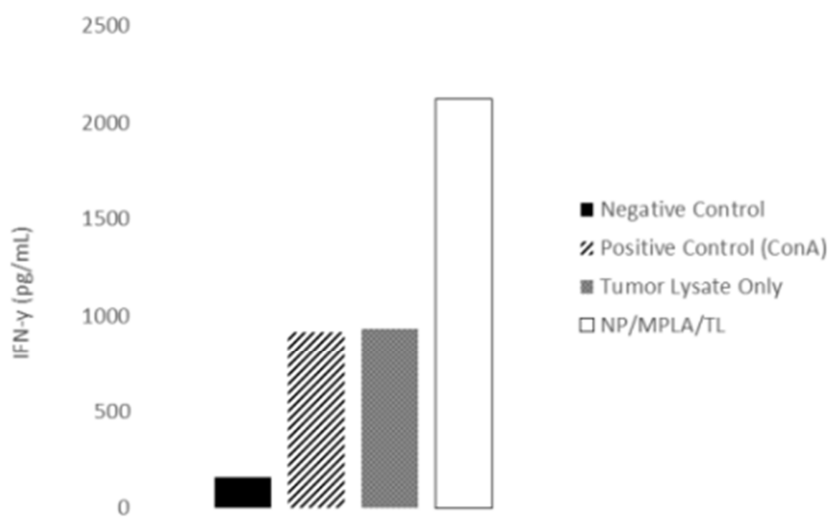

Figure 6. Cytokine profiling following dendritic cell/T cell stimulation was

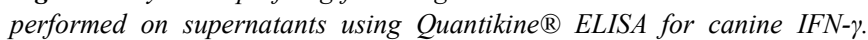
Nanoparticle-mediated antigen delivery resulted in increased expression of $I F N-\gamma$, which was not statistically significant $(p=0.338$ versus unencapsulated whole tumor lysate, and $p=0.159$ versus negative controls).
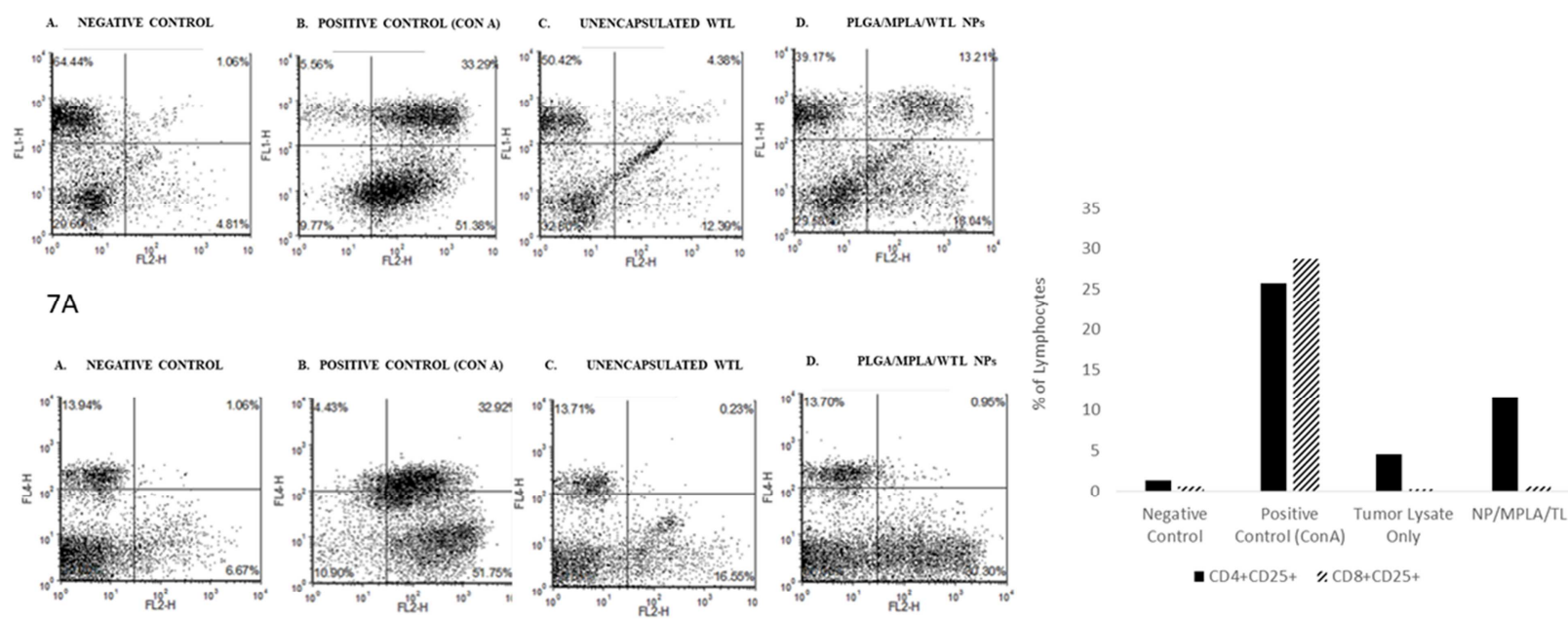

$7 \mathrm{~B}$

7C

Figure 7. A and B. Flow cytometric analysis of canine lymphocytes following completion of the dendritic cell/T cell co-culture (Day 12). A representative sample is shown. FL1-H (y-axis) and FL2-H (x-axis) were the channels used to assess CD4/CD25 (A) and CD8/CD25 (B) expression. Part C summarize findings of part $A$ and $B$. 


\section{Discussion}

The biologically aggressive behavior of canine MM poses a series of challenges to veterinarians and veterinary oncologists alike. Although it is not unique in its methods of immunoevasion, MM can manipulate the immune system in order to suppress anti-tumor activity and promote a microenvironment that is more conducive to its local and distant metastatic spread. Methods of immunoevasion include the synthesis and release of anti-inflammatory cytokines (i.e. IL-10; TGF- $\beta$ ), downregulation of $\beta 2$ microglobulin surface expression and therefore antigen presentation by APCs, and the stimulation of regulatory $\mathrm{T}$ cells, which can inhibit immune responses mediated by CD4+ and CD8+ lymphocytes [1, 6, 18]. In addition, aberrations in tyrosinase expression render current methods of treatment generally ineffective [6]. For these reasons, personalized immunotherapies are being explored, as they represent a means by which inherent mechanisms of tumor evasion can be overcome.

Dendritic cell-based vaccines are one means by which the cancer immunity cycle can be restored, and the dysfunctional, complex interactions between immune system and tumor can be restored [30]. DCs, which are considered natural adjuvants, effectively link the innate and adaptive arms of the immune system, and are therefore capable of enhancing immune responses, particularly when pulsed with peptide antigens [6, 18]. In the presented study, we generated an autologous DC vaccine prototype for canine patients with stages III-IV MM. The DCs were stimulated and activated ex vivo with PLGA NPs containing tumor-specific antigens and the immunestimulant MPLA. The vaccine was administered intradermally once a week for a series of 4 injections, and patients were serially monitored to evaluate for localized and systemic, adverse reactions. Circulating lymphocyte subsets were evaluated via flow cytometry pre- and post-vaccination, in addition to DC surface marker expression following activation with NP constructs.

Since DCs comprise $<1 \%$ of mononuclear cells in peripheral circulation, an accepted technique for the isolation and in vitro differentiation of DCs from monocytes was utilized for the generation of the described vaccine [37]. The incubation of monocytes with a standard cytokine cocktail of GM-CSF and IL-4 resulted in the successful generation of DCs in all sampled patients. Although an average of $1 \times 10^{6}$ DCs was administered to the patients each week, the number of mature DCs harvested was variable, with as few as $6.5 \times$ $10^{5}$ DCs administered on rare occasions. This is due to individual variation in the number of peripheral blood monocytes collected, and rates of cell death in culture. It is difficult to determine the clinical significance of this since a standard minimum number of DCs has not been defined for effective vaccination protocols. An increased volume of blood could potentially be collected from each patient to reduce these variations, as $7.5 \%$ of circulating blood volume may be safely collected once a week, or $15 \%$ through multiple samplings over a 2-week period [11]. However, estimated blood volume may be lower in sick or older patients, and obtaining greater volumes of whole blood may not be feasible, based on the patient's weight [11].

Following 6 days in culture, DCs were stimulated with PLGA NPs constructs containing antigens from a whole tumor lysate and MPLA. The NPs, which were generated using a double emulsion $\mathrm{W} / \mathrm{O} / \mathrm{W}$ solvent evaporation procedure, demonstrated mild size heterogeneity and minimal aggregation. Although mild variation was noted in their size, professional APCs are able to effectively uptake NPs up to $5 \mu \mathrm{m}$ via macro-pinocytosis or phagocytosis [13]. Therefore, heterogeneity in our NP size would not be a barrier to internalization by APC and effective antigen delivery was still anticipated. Successful stimulation and activation of the DCs was demonstrated by the increased expression of surface markers MHC I, MHC II and CD80 following 24 hours of incubation. Upregulation of these surface markers suggests effective maturation of the DCs, necessary for the activation of naïve CD4+ and CD8+ T cells in vivo.

Due to the heterogeneity of tumors and their aberrant expression of surface antigens (i.e. glycoproteins), a WTL was used for PLGA-NP synthesis. The lysate, which was obtained via repetitive freeze-thaw cycles, maximizes the number of tumor-associated antigens that can be used for stimulation of CD4+ and CD8+ T cells. Tumor-associated antigens, which include mutated and overexpressed antigens, are theorized to reduce the possibility of immunoevasion since immune responses are not limited to a narrow repertoire of antigens or epitopes [7]. Highly variable amounts of protein (ranging from 45.08 to $99.42 \mathrm{mg} / \mathrm{mL}$ ) were liberated from the treated tissues following the freezethaw process. The proteins, however, demonstrated similar banding patterns based on electrophoretic assays, suggesting that similar tumor-associated antigens may be released. Encapsulation efficiency averaged $53 \%$, and $>75 \%$ of the antigens were released over a 21-day period. As previously discussed, the delayed maximum release of antigen appreciated on Day 4 is unlikely to have negative clinical effects since the functional lifespan for mature DC antigen presentation is approximately 1 week [34]. Although previous studies have documented similar banding patterns between loaded and released proteins via SDS-PAGE, repeat assessment is required to ensure that the proteins have not denatured or aggregated during the encapsulation process specifically utilized by our laboratory. Such occurrences could alter the immunogenicity of the encapsulated antigens, and ultimately, render them therapeutically inactive [8].

The IFN- $\gamma$ assays and DC/T-cell co-culturing studies performed suggest that the proteins encapsulated in our NP formulations retain their antigenic activity and could be used to stimulate an adaptive immune response in vivo. Although not statistically significant, protein encapsulation within the PLGA-NPs containing MPLA resulted in a greater than twofold increase in the release of immunostimulatory IFN- $\gamma$ 
than WTL alone. When coupled with our previous report of enhanced pro-inflammatory cytokine (TNF- $\alpha$ and IL-12p40) and decreased immunoinhibitory IL-10 production, these findings support $\mathrm{T}$ cell stimulation. Based on the results of the flow cytometric analysis, however, the phenotypic profile of the activated $\mathrm{T}$ cells cannot be definitively concluded. The co-expression of $\mathrm{CD} 4+\mathrm{CD} 25+$ by small percentages of lymphocytes suggests either activated $\mathrm{T}$ helper cells (likely Th1 based on IFN- $\gamma$ production) or regulatory $\mathrm{T}$ cells. Additional intracellular (e.g. FoxP3) and surface markers are necessary for more definitive assessment of this particular population. The use of additional markers (e.g. CD21, CD79a, CD56) may also help identify the double negative population of lymphocytes present, which may represent activated B cells, NK cells, or DCs.

Although 3 of the enrolled patients experienced localized adverse events following intradermal vaccination, the vaccine was well-tolerated overall, with minimal side effects observed. No evidence of systemic toxicity was identified based on serial hematologic and biochemical analyses. These findings corroborate previously published studies demonstrating the overall safety of PLGA NPs and DC vaccinations at large. The long-term, anti-tumor effects of our vaccine cannot be properly evaluated due to the small number of canines utilized, and the lack of a randomized control group. However, none of the patients showed an objective response in tumor size, and progressive disease was documented in all by the end of the trial. Despite these findings, it is important to note that the majority of patients were in the late stages of disease, with evidence of local and/or systemic metastases and a high tumor burden. In addition, several patients had previously failed other adjunctive therapies (i.e. radiation therapy; Oncept ${ }^{\circledR}$ ), and therefore, could have had multiple inhibitory, immunoevasive mechanisms in place. For these reasons, it is difficult to speculate as to the true potential of our NP-based DC vaccine. These case studies, however, emphasize the importance of achieving localized tumor control prior to the initiation of adjunctive therapies.

\section{Conclusion}

In summary, PLGA/MPLA nanoparticle constructs biochemical characterization; encapsulation efficiency and kinetic release were determined. Results showed that dog melanoma tumor antigens were effectively incorporated in the nanoparticle constructs. In addition, nanoparticle constructs effectively activated autologous DCs, and induced release of the pro-immune cytokine IFN- $\gamma$. No significant adverse effects were observed in any of the patients, and flow cytometry of whole blood revealed increased CD4:CD8 T lymphocyte ratios. These results suggest that a $\mathrm{DC}$ vaccine utilizing PLGA/MPLA nanoparticle technology initiates adaptive immune responses and is safe to administer to canine although a larger cohort of patients is warranted. Our future strategies therefore include the assessment of our DC vaccine as an adjunctive therapy following surgical excision of the primary tumor, in a randomized clinical trial that includes canines with earlier stages of disease. Since the effective control of MM and other cancers will likely require a multimodal approach, our future strategies also include the use of T cell checkpoint inhibitors, such as CTLA-4, PD-1 and PD-L1. Antibodies against these immune checkpoint molecules can theoretically result in an enhanced anti-tumor response by reactivating anergic cytotoxic $\mathrm{T}$ cells. Use of monoclonal antibodies against these molecules has already proven beneficial in human cases of $\mathrm{MM}$, and recently published studies revealed limited, positive clinical results in canine cases, as well $[16,22]$. The combination of such inhibitors with a NP-based DC vaccine is the next phase in the search for an effective form of immunotherapy that is capable of halting the aggressive tumor phenotype.

\section{Conflict of Interest}

The authors declare no conflict of interest.

\section{Acknowledgements}

This research was supported by Marge Crowley Canine Research Endowment (grant \#9839) and an intramural grant (\#4809) from Washington State University College of Veterinary Medicine. We thank Dr. William C. Davis, Dr. Gaber S. Abdellrazeq, and Maria Clara de Souza for their assistance with this research.

\section{References}

[1] Atherton MJ, Morris JS, McDermott MR, and Lichty BD. Cancer immunology and canine malignant melanoma: A comparative review. Veterinary Immunol Immunopathol 2016, $169,15-26$.

[2] Basch E, Reeve BB, Mitchell SA, Clauser SB, Minasian LM, Dueck AC, Mendoza TR, Hay J, Atkinson TM, Abernethy AP, Bruner DW, Cleeland CS, Sloan JA, Chilukuri R, Baumgartner P, Denicoff A, St. Germain D, O'Mara AM, Chen A, Kelaghan J, Bennett AV, Sit L, Rogak L, Barz A, Paul DB and Schrag D. Development of the National Cancer Institute's Patient-Reported Outcomes Version of the Common Terminology Criteria for Adverse Events (PRO-CTCAE). JNCI 2014, 106, 1-11.

[3] Bergman PJ, Camps-Palau MA, McKnight JA, Leibman NF, Craft DM, Leung C, Liao J, Riviere I, Sadelain M, Hohenhaus AE, Gregor P, Houghton AN, Perales MA, and Wolchok JD. Development of a xenogeneic DNA vaccine program for canine malignant melanoma at the Animal Medical Center. Vaccine 2006, 24, 4582-4585.

[4] Boston SE, Lu X, Culp WT, Montinaro V, Romanelli G, Dudley RM, Liptak JM, Mestrinho LA, Buracco P. Efficacy of systemic adjuvant therapies administered to dogs after excision of oral malignant melanomas: 151 cases (2001-2012). JAVMA 2014, 245, 401-407.

[5] Brockley LK, Cooper MA and Bennett PF. Malignant melanoma in 63 dogs (2001-2011): the effect of carboplatin chemotherapy on survival. NZVJ 2013, 61, 25-31. 
[6] Butterfield LH. Dendritic cells in cancer immunotherapy clinical trials: are we making progress? Front Immunol 2013, $4,1-7$.

[7] Chiang CL, Benencia F, and Coukos G. Whole Tumor Antigen Vaccines. Semin Immunol 2010, 22, 132-143.

[8] Danhier F, Ansorena E, Silva JM, Coco R, Le Breton A, and Preat V. PLGA-based nanoparticles: An overview of biomedical applications. J Control Release 2012, 161, 505-522.

[9] Elamanchili P, Lutsiak CME, Hamdy S, Diwan M, and Samuel J. "Pathogen-Mimicking" Nanoparticles for Vaccine Delivery to Dendritic Cells. J Immunother 2007, 30, 378-395.

[10] Fan Y, and Moon JJ. Nanoparticle Drug Delivery Systems Designed to Improve Cancer Vaccines and Immunotherapy. Vaccines 2015, 3, 662-685.

[11] Field G and Jackson TA. 2007. The Laboratory Canine, 1st ed. Taylor \& Francis, Florida.

[12] Grosenbaugh DA, Leard AT, Bergman PJ, Klein MK, Meleo K, Susaneck S, Hess PR, Jankowski MK, Jones PD, Leibman NF, Johnson MH, Kurzman ID, and Wolchok JD. Safety and efficacy of a xenogeneic DNA vaccine encoding for human tyrosinase as adjunctive treatment for oral malignant melanoma in dogs following surgical excision of the primary tumor. AJVR 2011, 72, 1631-1638.

[13] Guldner D, Hwang, JK, Cardieri MCD, Eren M, Ziaei P, Norton MG, and Souza CD. In Vitro Evaluation of the Biological Responses of Canine Macrophages Challenged with PLGA Naoparticles Containing Monophosphoryl Lipid A. PloS ONE 2016, 11, 1-20.

[14] Gutzwiller MER, Moulin HR, Zurbriggen A, Roosje P, and Summerfield A. Comparative analysis of canine monocyte- and bone-marrow-derived dendritic cells. Vet Res 2010, 41, 1-12.

[15] Hamdy S, Haddadi A, Shayeganpour A, Samuel J and Lavasanifar A. Activation of antigen-specific T cell-responses by mannan-decorated PLGA nanoparticles. Pharm Res 2011, $28,2288-2301$.

[16] Hernandez B, Adissu HA, Wei BR, Michael HT, Merlino G, and Simpson RM. Naturally Occurring Canine Melanoma as a Predictive Comparative Oncology Model for Human Mucosal and Other Triple Wild-Type Melanomas. Int J Mol Sci 2018, 19, 1-19.

[17] Hobart M, Ramassar V, Goes N, Urmson J, and Halloran PF. IFN regulatory factor-1 plays a central role in the regulation of the expression of class I and II MHC genes in vivo. J Immol $1997,158,4260-4269$.

[18] Horiuchi Y, Tominaga M, Ichikawa M, Yamashita M, Okano K, Jikumaru Y, Nariai Y, Nakajima Y, Kuwabara M, and Yukawa M. Relationship between regulatory and type $1 \mathrm{~T}$ cells in dogs with oral malignant melanoma. Microbiol Immunol 2010, 54, 152-159.

[19] Klippstein R and Pozo D. Nanotechnology-based manipulation of dendritic cells for enhanced immunotherapy strategies. Nanomedicine 2010, 6, 523-529.

[20] MacEwen EG, Patnaik AK, Harbey HJ, Hayes AA and Matus R. Canine oral melanoma: comparison of surgery versus surgery plus Corynebacterium parvum. Cancer Invest 1986, 4, 397-402.

[21] MacEwen EG, Kurzman ID, Vail DM, Dubielziq RR, Everlith
K, Mdewell BR, Rodriguez CO Jr, Phillips B, Zwahlen CH, Obradovich J, Rosenthal RC, Fox LE, Rosenberg M, Henry C, and Fidel J. Adjuvant therapy for melanoma in dogs: results of randomized clinical trials using surgery, liposome-encapsulated muramyl-tripeptide, and granulocyte macrophage colonystimulating factor. Clin Cancer Res 1999, 5, 4249-4258.

[22] Maekawa N, Konnai S, Takagi S, Kagawa Y, Okagawa T, Nishimori A, Ikebuchi R, Izumi Y, Deguchi T, Nakajima C, Kato Y, Yamamoto K, Uemura H, Suzuki Y, Murata S, and Ohashi K. A canine chimeric monoclonal antibody targeting PD-L1 and its clinical efficacy in canine oral malignant melanoma or undifferentiated sarcoma. Sci Rep 2017, 7, 1-12.

[23] Meyer A, Gruber AD and Klopfleisch R. CD25 Is expressed by canine cutaneous mast cell tumors but not by cutaneous connective tissue mast cells. Vet Pathol 2012, 49, 988-997.

[24] Nakai N, Hartmann G, Kishimoto S, and Katoh N. Dendritic cell vaccination in human melanoma: relationships between clinical effects and vaccine parameters. Pigment Cell Melanoma Res 2010, 23, 607-619.

[25] O'Connor CM and H. Wilson-Robles. Developing T Cell Cancer Immunotherapy in the Dog with Lymphoma. ILAR J 2014, 55, 169-181.

[26] O'Neill K, Guth A, Biller B, Elmslie R, and Dow S. Changes in regulatory $\mathrm{T}$ cells in dogs with cancer and association with tumor type. JVIM 2009, 23, 875-881.

[27] Ottnod JM, Smedley RC, Walshaw R, Hauptman JG, Kiupel $\mathrm{M}$ and Obradovich JE. A retrospective analysis of the efficacy of Oncept vaccine for the adjunct treatment of canine oral malignant melanoma. Vet Comp Oncol 2013, 11, 219-229.

[28] Prasad S, Cody V, Saucier-Sawyre JK, Saltzman WM, Sasaki CT, Edelson RL, Birchall MA, and Hanlon DJ. Polymer nanoparticles containing tumor lysates as antigen delivery vehicles for dendritic cell-based anti-tumor immunotherapy. Nanomedicine 2011, 7, 1-10.

[29] Reis e Sousa C. Activation of dendritic cells: translating innate into adaptive immunity. Curr Opin Immunol 2004, 16, 21-25.

[30] Sahin U and Türeci . Personalized vaccines for cancer immunotherapy. Science 2018, 359, 1355-1360.

[31] Salcedo M, Bercovici N, Taylor R, Vereecken P, Massicard S, Duriau D, Vernel-Pauillac F, Boyer A, Baron-Bodo V, Mallard E, Bartholeyns J, Goxe B, Latour N, Leroy S, Prigent D, Martiat P, Sales F, Laporte M, Bruyns C, Romet-Lemonne JL, Abastado JP, Lehmann $F$ and Velu T. Vaccination of melanoma patients using dendritic cells loaded with an allogeneic tumor cell lysate. Cancer Immunol Immunother 2006, 55, 819-829.

[32] Sarti F, Perera G, Hintzen F, Kotti K, Krageorgiou V, Kammona O, Kiparissides C, and Bernkop-Schnurch A. In vivo evidence of oral vaccination with PLGA nanoparticles containing the immunostimulant monophosphoryl lipid A. Biomaterials 2011, 32, 4052-4057.

[33] Smedley RC, Lamoureux J, Sledge DG and Kiupel M. Immunohistochemical diagnosis of canine oral amelanotic melanocytic neoplasms. Veterinary Pathology 2011, 48, 32-40.

[34] Solbrig CM, Saucier-Sawyer JK, Cody V. Saltzman WM, and Hanlon DJ. Polymer nanoparticles for immunotherapy from encapsulated tumor-associated antigens and whole tumor cells. Mol Pharm 2007, 4, 47-57. 
[35] Tamura K, Yamada M, Isotani M, Arai H, Yagihara H, Ono K, Washizu T, and Bonkobara M. Induction of dendritic cellmediated immune responses against canine malignant melanoma cells. Vet J 2008, 175, 126-129.

[36] Treggiari E, Grant, JP and North SM. A retrospective review of outcome and survival following surgery and adjuvant xenogeneic DNA vaccination in 32 dogs with oral malignant melanoma. J Vet Med Sci 2016, 78, 845-850.

[37] Trepiakas R, Berntsen A, Hadrup SR, Bjorn J, Geertsen PF, Straten PT, Andersen MH, Pedersen AE, Soleimani A, Lorentzen T, Johansen JS, and Svane IM. Vaccination with autologous dendritic cells pulsed with multiple tumor antigens for treatment of patients with malignant melanoma: results from a phase I/II trial. Cytotherapy 2010, 12, 721-734.

[38] Uto T, Akagi T, Hamasaki T, Akashi M and Baba M. Modulation of innate and adaptive immunity by biodegradable nanoparticles. Immunol Lett 2009, 125, 46-52.

[39] Waeckerle-Men Y and Groettrup M. PLGA microspheres for improved antigen delivery to dendritic cells as cellular vaccines. Adv Drug Deliv Rev 2005, 57, 475-482.

[40] Withrow SJ and Vail DM. 2007. Withrow \& MacEwen's Small Animal Clinical Oncology, 4th ed. Saunders, Missouri. 